\title{
ON EISENSTEIN IDEALS AND THE CUSPIDAL GROUP OF $J_{0}(N)$
}

\author{
HWAJONG YOO
}

\begin{abstract}
Let $\mathcal{C}_{N}$ be the cuspidal subgroup of the Jacobian $J_{0}(N)$ for a square-free integer $N>6$. For any Eisenstein maximal ideal $\mathfrak{m}$ of the Hecke ring of level $N$, we show that $\mathcal{C}_{N}[\mathfrak{m}] \neq 0$. To prove this, we calculate the index of an Eisenstein ideal $\mathcal{I}$ contained in $\mathfrak{m}$ by computing the order of the cuspidal divisor annihilated by $\mathcal{I}$.
\end{abstract}

\section{CONTENTS}

1. Introduction 1

2. Eisenstein ideals 2

3. The cuspidal group 3

4. Eisenstein series $\quad 6$

5. The index of an Eisenstein ideal $\quad 7$

6. Proof of the main theorem 10

$\begin{array}{lr}\text { References } & 10\end{array}$

\section{INTRODUCTION}

Let $N$ be a square-free integer greater than 6 and let $X_{0}(N)$ denote the modular curve over $\mathbb{Q}$ associated to $\Gamma_{0}(N)$, the congruence subgroup of $\mathrm{SL}_{2}(\mathbb{Z})$ consisting of upper triangular matrices modulo $N$. There is the Hecke ring $\mathbb{T}:=\mathbb{T}(N)$ of level $N$, which is the subring of the endomorphism ring of the Jacobian variety $J_{0}(N):=$ $\operatorname{Pic}^{0}\left(X_{0}(N)\right)$ of $X_{0}(N)$ generated by the Hecke operators $T_{n}$ for all $n \geq 1$. A maximal ideal $\mathfrak{m}$ of $\mathbb{T}$ is called Eisenstein if the two dimensional semisimple representation $\rho_{\mathfrak{m}}$ of $\operatorname{Gal}(\overline{\mathbb{Q}} / \mathbb{Q})$ over $\mathbb{T} / \mathfrak{m}$ attached to $\mathfrak{m}$ is reducible, or equivalently $\mathfrak{m}$ contains the ideal

$$
\mathcal{I}_{0}(N):=\left(T_{r}-r-1: \text { for primes } r \nmid N\right) .
$$

Let $\mathcal{C}_{N}$ be the cuspidal group of $J_{0}(N)$ generated by degree 0 cuspidal divisors, which is finite by Manin and Drinfeld [11,4].

Ribet conjectured that all Eisenstein maximal ideals are "cuspidal". In other words, $\mathcal{C}_{N}[\mathfrak{m}] \neq 0$ for any Eisenstein maximal ideal $\mathfrak{m}$. There were many evidences of this conjecture. In particular, special cases were already known (cf. [21, §3]). In this paper, we prove his conjecture.

Theorem 1.1 (Main theorem). Let $\mathfrak{m}$ be an Eisenstein maximal ideal of $\mathbb{T}$. Then $\mathcal{C}_{N}[\mathfrak{m}] \neq 0$.

To prove this theorem, we classify all possible Eisenstein maximal ideals in $\S 2$. From now on, we denote by $U_{p}$ the $p^{\text {th }}$ Hecke operator $T_{p} \in \mathbb{T}$ when $p \mid N$.

Proposition 1.2. Let $\mathfrak{m}$ be an Eisenstein maximal ideal of $\mathbb{T}$. Then, it contains

$$
I_{M, N}:=\left(U_{p}-1, U_{q}-q, \mathcal{I}_{0}(N): \text { for primes } p \mid M \text { and } q \mid N / M\right)
$$

for some divisor $M$ of $N$ such that $M \neq 1$.

2010 Mathematics Subject Classification. 11G18, $14 \mathrm{G} 35$.

Key words and phrases. Eisenstein ideals, Cuspidal groups. 
In $\S 3$, we study basic properties of the cuspidal group $\mathcal{C}_{N}$ of $J_{0}(N)$. In particular, we explicitly compute the order of the cuspidal divisor $C_{M, N}$, which is the equivalence class of $\sum_{d \mid M}(-1)^{\omega(d)} P_{d}$, where $\omega(d)$ is the number of distinct prime divisors of $d$ and $P_{d}$ is the cusp of $X_{0}(N)$ corresponding to $1 / d \in \mathbb{P}^{1}(\mathbb{Q})$.

Theorem 1.3. The order of $C_{M, N}$ is equal to the numerator of $\frac{\varphi(N) \psi(N / M)}{24} \times h$, where $h$ is either 1 or 2 . Moreover, $h=2$ if and only if one of the following holds:

(1) $N=M$ and $M$ is a prime such that $M \equiv 1(\bmod 8)$;

(2) $N=2 M$ and $M$ is a prime such that $M \equiv 1(\bmod 8)$.

(See Notation 1.1 for the definition of $\varphi(N)$ and $\psi(N)$.) This theorem generalizes the works by Ogg $[14,15]$ and Chua-Ling [1] to the case where $\omega(N) \geq 3$. In $\S 4$, we introduce Eisenstein series and compute their residues at various cusps. With these computations, we can prove the following theorem in $\S 5$.

Theorem 1.4. If $M \neq N$ and $N / M$ is odd, then the index of $I_{M, N}$ is equal to the order of $C_{M, N}$. Moreover, if $M=N$ or $N / M$ is even, then the index of $I_{M, N}$ is equal to the order of $C_{M, N}$ up to powers of 2.

Finally, combining all the results above, we prove our main theorem in $\S 6$.

Acknowledgements. We are grateful to Ken Ribet for suggesting the problem and his advice during the preparation of this work. We thank the anonymous referee for careful reading and a number of suggestions and corrections to improve the paper.

1.1. Notation. For a square-free integer $N=\prod_{i=1}^{n} p_{i}$, we define the following quantities:

$$
\begin{gathered}
\omega(N):=n=\text { the number of distinct prime divisors of } N ; \\
\varphi(N):=\prod_{i=1}^{n}\left(p_{i}-1\right) \quad \text { and } \quad \psi(N):=\prod_{i=1}^{n}\left(p_{i}+1\right) .
\end{gathered}
$$

For any rational number $x=a / b$, we denote by num $(x)$ the numerator of $x$, i.e.,

$$
\operatorname{num}(x):=\frac{a}{(a, b)} .
$$

For a prime divisor $p$ of $N$, there is the degeneracy map $\gamma_{p}: J_{0}(N / p) \times J_{0}(N / p) \rightarrow J_{0}(N)$ (cf. [16, $\left.\S 3\right]$ ). The image of $\gamma_{p}$ is called the p-old subvariety of $J_{0}(N)$ and is denoted by $J_{0}(N)_{p \text {-old }}$. The quotient of $J_{0}(N)$ by $J_{0}(N)_{p \text {-old }}$ is called the $p$-new quotient and is denoted by $J_{0}(N)^{p \text {-new }}$. Note that $J_{0}(N)_{p \text {-old }}$ is stable under the action of Hecke operators and $\gamma_{p}$ is Hecke-equivariant. Accordingly, the image of $\mathbb{T}(N)$ in $\operatorname{End}\left(J_{0}(N)_{p \text {-old }}\right)$

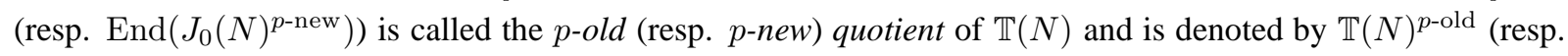
$\mathbb{T}(N)^{p \text {-new }}$ ). A maximal ideal $\mathfrak{m}$ of $\mathbb{T}(N)$ is called $p$-old (resp. $p$-new) if its image in $\mathbb{T}(N)^{p \text {-old }}$ (resp. $\mathbb{T}(N)^{p \text {-new })}$ is still maximal. Note that if a maximal ideal $\mathfrak{m}$ of $\mathbb{T}(N)$ is $p$-old, then there is a maximal ideal $\mathfrak{n}$ of $\mathbb{T}(N / p)$ corresponding to $\mathfrak{m}$ (cf. [17, §7]).

For a prime divisor $p$ of $N$, we denote by $w_{p}$ the Atkin-Lehner operator (with respect to $p$ ) acting on $J_{0}(N)$ (and the space of modular forms of level $N$ ). (For more detail, see [13, §1].)

For a prime $p$, we denote by $\operatorname{Frob}_{p}$ an arithmetic Frobenius element for $p$ in $\operatorname{Gal}(\overline{\mathbb{Q}} / \mathbb{Q})$.

\section{EISENSTEIN IDEALS}

From now on, we denote by $N$ a square-free integer greater than 6 and let $\mathbb{T}:=\mathbb{T}(N)$ be the Hecke ring of level $N$. A maximal ideal $\mathfrak{m}$ of $\mathbb{T}$ is called Eisenstein if the two dimensional semisimple representation $\rho_{\mathfrak{m}}$ of $\operatorname{Gal}(\overline{\mathbb{Q}} / \mathbb{Q})$ over $\mathbb{T} / \mathfrak{m}$ attached to $\mathfrak{m}$ is reducible, or equivalently $\mathfrak{m}$ contains the ideal $\mathcal{I}_{0}(N):=\left(T_{r}-r-1:\right.$ for primes $\left.r \nmid N\right)$. (For the existence of $\rho_{\mathfrak{m}}$, see [17, Proposition 5.1].)

Let us remark briefly why these two definitions are equivalent. Let $\mathfrak{m}$ be a maximal ideal of $\mathbb{T}$ containing $\ell$. If $\rho_{\mathfrak{m}}$ is reducible, then $\rho_{\mathfrak{m}} \simeq \mathbb{1} \oplus \chi_{\ell}$, where $\mathbb{1}$ is the trivial character and $\chi_{\ell}$ is the mod $\ell$ cyclotomic character, by Ribet [22, Proposition 2.1]. Therefore for a prime $r$ not dividing $\ell N$, we have

$$
T_{r}(\bmod \mathfrak{m})=\operatorname{trace}\left(\rho_{\mathfrak{m}}\left(\operatorname{Frob}_{r}\right)\right)=1+r
$$


and hence $T_{r}-r-1 \in \mathfrak{m}$. For $r=\ell$, we get $T_{\ell} \equiv 1+\ell \equiv 1(\bmod \mathfrak{m})$ by Ribet [18, Lemma 1.1]. (This lemma basically follows from the result by Deligne [5, Theorem 2.5] and this is also true even when $\ell$ divides $N$.) Conversely, if $\mathfrak{m}$ contains $\mathcal{I}_{0}(N)$, then $\rho_{\mathfrak{m}} \simeq \mathbb{1} \oplus \chi_{\ell}$ by the Chebotarev and the Brauer-Nesbitt theorems.

To classify all Eisenstein maximal ideals, we need to understand the image of $U_{p}$ in the residue fields for any prime divisor $p$ of $N$.

Lemma 2.1. Let $\mathfrak{m}$ be an Eisenstein maximal ideal of $\mathbb{T}$. Let $p$ be a prime divisor of $N$ and $U_{p}-\epsilon(p) \in \mathfrak{m}$. Then, $\epsilon(p)$ is either 1 or $p$ modulo $\mathfrak{m}$.

Proof. Assume that $\mathfrak{m}$ is $p$-old. Then $\mathfrak{m}$ can be regarded as a maximal ideal of $\mathbb{T}^{p \text {-old }}$. Let $R$ be the common subring of the Hecke ring $\mathbb{T}(N / p)$ of level $N / p$ and $\mathbb{T}^{p \text {-old }}$, which is generated by all $T_{n}$ with $p \nmid n$. Let $\mathfrak{n}$ be the corresponding maximal ideal of $\mathbb{T}(N / p)$ to $\mathfrak{m}$ and $T_{p}$ be the $p^{\text {th }}$ Hecke operator in $\mathbb{T}(N / p)$. Then, we get

$$
\mathbb{T}(N / p)=R\left[T_{p}\right] \quad \text { and } \quad \mathbb{T}(N)^{p \text {-old }}=R\left[U_{p}\right]
$$

$[17, \S 7]$ and $\mathbb{T} / \mathfrak{m} \simeq \mathbb{T}(N / p) / \mathfrak{n}$. Two operators $T_{p}$ and $U_{p}$ are connected by the quadratic equation $U_{p}^{2}-T_{p} U_{p}+p=$ 0 (loc. cit.). Note that $T_{p}-p-1 \in \mathfrak{n}$ because $\mathfrak{n}$ is Eisenstein as well. Therefore over the ring $\mathbb{T} / \mathfrak{m} \simeq \mathbb{T}(N / p) / \mathfrak{n}$, we get $U_{p}^{2}-(p+1) U_{p}+p=\left(U_{p}-1\right)\left(U_{p}-p\right)=0$ and hence either $\epsilon(p) \equiv 1$ or $p(\bmod \mathfrak{m})$.

Assume that $\mathfrak{m}$ is $p$-new. Then $\epsilon(p)= \pm 1$. Therefore it suffices to show that $\epsilon(p) \equiv 1$ or $p(\bmod \mathfrak{m})$ when $\epsilon(p)=$ -1 . Let $\ell$ be the residue characteristic of $\mathfrak{m}$. If $\ell=2$, then there is nothing to prove because $1 \equiv-1(\bmod \mathfrak{m})$. If $\ell=p$, then $U_{p} \equiv 1(\bmod \mathfrak{m})$ by Ribet $[18$, Lemma 1.1$]$. Therefore we assume that $\ell \geq 3$ and $\ell \neq p$. On the one hand, we have $\rho_{\mathfrak{m}} \simeq \mathbb{1} \oplus \chi_{\ell}$. On the other hand, the semisimplification of the restriction of $\rho_{\mathfrak{m}} \operatorname{to} \operatorname{Gal}\left(\overline{\mathbb{Q}}_{p} / \mathbb{Q}_{p}\right)$ is isomorphic to $\epsilon \oplus \epsilon \chi_{\ell}$, where $\epsilon$ is the unramified quadratic character with $\epsilon\left(\operatorname{Frob}_{p}\right)=\epsilon(p)$ because $\mathfrak{m}$ is $p$-new (cf. [2, Theorem 3.1.(e)]). Since $\epsilon(p)=-1$, we get $p \equiv-1(\bmod \ell)$ and hence $\epsilon(p) \equiv p(\bmod \mathfrak{m})$.

Let $\mathfrak{m}$ be an Eisenstein maximal ideal of $\mathbb{T}$ containing $\ell$. Then, it contains

$$
I_{M, N}:=\left(U_{p}-1, U_{q}-q, \mathcal{I}_{0}(N): \text { for primes } p \mid M \text { and } q \mid N / M\right) \subseteq \mathbb{T}
$$

for some divisor $M$ of $N$ by the previous lemma. If $q \equiv 1(\bmod \ell)$ for a prime divisor $q$ of $N / M$, then $\mathfrak{m}=$ $\left(\ell, I_{M, N}\right)=\left(\ell, I_{M \times q, N}\right)$. Therefore when we denote by $\mathfrak{m}:=\left(\ell, I_{M, N}\right)$ for some divisor $M$ of $N$, we always assume that $q \not \equiv 1(\bmod \ell)$ for all prime divisors $q$ of $N / M$. Hence if $\ell=2$, then either $\mathfrak{m}:=\left(\ell, I_{N, N}\right)$ or $\mathfrak{m}:=\left(\ell, I_{N / 2, N}\right)$. If $\ell \geq 3, \mathfrak{m}:=\left(\ell, I_{1, N}\right)$ cannot be maximal by Proposition 5.5. Therefore from now on, we always assume that $M \neq 1$.

\section{THE CUSPIDAL GROUP}

As before, let $N$ denote a square-free integer and let $M \neq 1$ denote a divisor of $N$. For a divisor $d$ of $N$, we denote by $P_{d}$ the cusp corresponding to $1 / d$ in $\mathbb{P}^{1}(\mathbb{Q})$. (Thus, the cusp $\infty$ is denoted by $P_{N}$.) We denote by $C_{M, N}$ the equivalence class of a cuspidal divisor $\sum_{d \mid M}(-1)^{\omega(d)} P_{d}$. Note that $I_{M, N}$ annihilates $C_{M, N}$ [21, Proposition 2.13]. To compute the order of $C_{M, N}$, we use the method of Ling [10, $\left.\S 2\right]$.

Theorem 3.1. The order of $C_{M, N}$ is equal to

$$
\operatorname{num}\left(\frac{\varphi(N) \psi(N / M)}{24}\right) \times h,
$$

where $h$ is either 1 or 2 . Moreover, $h=2$ if and only if one of the following holds:

(1) $N=M$ and $M$ is a prime such that $M \equiv 1(\bmod 8)$;

(2) $N=2 M$ and $M$ is a prime such that $M \equiv 1(\bmod 8)$.

Remark 3.2. The size of the set $\mathcal{C}_{N}$ is computed by Takagi [19]. Recently, Harder discussed the more general question of giving denominators of Eisenstein cohomology classes. The order of a cuspidal divisor is a special case of such a denominator and some cases were computed by a slightly different method from the one used here [7, $\S 2]$. 
Before starting to prove this theorem, we define some notations and provide lemmas.

Let $N=\prod_{i=1}^{n} p_{i}$. We denote by $\mathcal{S}$ the set of divisors of $N$. Let $s:=2^{n}=\# \mathcal{S}$.

(1) For $a \in \mathcal{S}$, we denote by

$$
a=\left(a_{1}, a_{2}, \cdots, a_{n}\right),
$$

where $a_{i}=0$ if $\left(p_{i}, a\right)=1$; and $a_{i}=1$ otherwise. For instance, $1=(0,0, \cdots, 0)$ and $N=$ $(1,1, \cdots, 1)$.

(2) We define the total ordering on $\mathcal{S}$ as follows.

Let $a, b \in \mathcal{S}$ and $a \neq b$.

- If $\omega(a)<\omega(b)$, then $a<b$. In particular, $1<a<N$ for $a \in \mathcal{S} \backslash\{1, N\}$.

- If $\omega(a)=\omega(b)$, then we use the anti-lexicographic order. In other words, $a<b$ if $a_{i}=b_{i}$ for all $i<t$ and $a_{t}>b_{t}$.

(3) We define the box addition $\boxplus$ on $\mathcal{S}$ as follows.

$$
a \boxplus b:=\left(c_{1}, c_{2}, \cdots, c_{n}\right),
$$

where $c_{i} \equiv a_{i}+b_{i}+1(\bmod 2)$ and $c_{i} \in\{0,1\}$. For instance, $p_{1} \boxplus p_{1}=N$ and $1 \boxplus a=N / a$.

(4) Finally, we define the sign on $\mathcal{S}$ as follows.

$$
\operatorname{sgn}(a):=(-1)^{s(a)}
$$

where $s(a)=\omega(N)-\omega(a)$. For example, $\operatorname{sgn}(N)=1$ and $\operatorname{sgn}(1)=(-1)^{n}$.

We denote by $\mathcal{S}=\left\{d_{1}, d_{2}, \ldots, d_{s}\right\}$, where $d_{i}<d_{j}$ if $i<j$. For instance, $d_{1}=1, d_{2}=p_{1}$ and $d_{s}=N$. Note that $d_{i} \times d_{s+1-i}=N$ for any $i$.

For ease of notation, we denote by $d_{i j}$ the box sum $d_{i} \boxplus d_{j}$.

Lemma 3.3. We have the following properties of $\boxplus$.

(1) $d_{i j}=d_{j i}=d_{s+1-i} \boxplus d_{s+1-j}$.

(2) $d_{i 1}=N / d_{i}=d_{s+1-i}$.

(3) $\mathcal{S}=\left\{d \boxplus d_{1}, d \boxplus d_{2}, \ldots, d \boxplus d_{s}\right\}$ for any $d=d_{i}$.

(4) $\operatorname{sgn}\left(d_{i j}\right)=\operatorname{sgn}\left(d_{i}\right) \times \operatorname{sgn}\left(d_{j}\right)$.

(5) Assume that $i \neq j$ and $d_{i j}$ is not divisible by $p_{n}$. Then, for any $d_{k}$ such that $d_{k j}$ is not divisible by $p_{n}$, we get

$$
d_{i k} \times d_{k j}=d_{i r(k)} \times d_{r(k) j},
$$

where $r(k)$ is the unique integer between 1 and such that $d_{r(k) j}=p_{n} \cdot d_{k j}$.

Proof. The first, second, third and fourth assertions easily follow from the definition.

Assume that $i \neq j$. Then $d_{i j} \neq N$ and there is a prime divisor of $N / d_{i j}$. Assume that $d_{i j}$ is not divisible by $p_{n}$. Let $k$ be an integer such that $d_{k j}$ is not divisible by $p_{n}$. Then, we denote by

$$
\begin{aligned}
d_{i} & =\left(a_{1}, \cdots, a_{n}\right) \quad \text { and } \quad d_{j}=\left(b_{1}, \cdots, b_{n}\right) ; \\
d_{k} & =\left(c_{1}, \cdots, c_{n}\right) \quad \text { and } \quad d_{r(k)}=\left(e_{1}, \cdots, e_{n}\right) .
\end{aligned}
$$

By abuse of notation, we denote by $d_{i k} \times d_{k j}=\left(x_{1}, x_{2}, \cdots, x_{n}\right)$ and $d_{i r(k)} \times d_{r(k) j}=\left(y_{1}, y_{2}, \cdots, y_{n}\right)$, where $0 \leq x_{t}, y_{t} \leq 2$. Thus, $d_{i k} \times d_{k j}=\prod_{t=1}^{s} p_{t}^{x_{t}}$. It suffices to show that $x_{t}=y_{t}$ for all $t$.

- Assume that $t \neq n$. From the definition of $d_{r(k)}$, we get $c_{t}=e_{t}$. Therefore $x_{t}=y_{t}$.

- Since $d_{i j}$ and $d_{k j}$ is not divisible by $p_{n}$, we get $a_{n}+b_{n}=1=c_{n}+b_{n}$. Therefore $a_{n}=c_{n}$. Since $d_{r(k) j}$ is divisible by $p_{n}$, we get $e_{n}+b_{n}+1 \equiv 1(\bmod 2)$. Therefore $x_{n}=y_{n}=1$.

From now on, we follow the notations in $[10, \S 2]$. In our case, the $s \times s$ matrix $\Lambda$ on page 35 of op. cit. is of the form

$$
\Lambda_{i j}=\frac{1}{24} a_{N}\left(d_{i}, d_{j}\right)
$$


where

For examples, $a_{N}(1, p)=N / p$ and $a_{N}(N, p)=p$.

$$
a_{N}(a, b):=\frac{N}{(a, N / a)} \frac{(a, b)^{2}}{a b} .
$$

Lemma 3.4. We get

$$
24 \times \Lambda_{i j}=d_{i} \boxplus d_{j}=d_{i j} \in \mathcal{S} .
$$

Proof. This is clear from the definition.

Lemma 3.5. Let $A:=\left(\operatorname{sgn}\left(d_{i j}\right) \times\left(d_{i j}\right)\right)_{1 \leq i, j \leq s}$ be a $s \times s$ matrix. Then, $A=\frac{\varphi(N) \psi(N)}{24} \times \Lambda^{-1}$.

Proof. We compute $B:=24 \times \Lambda \times A$.

- Assume that $i=j$. Then, we have

$$
B_{i i}=\sum_{j=1}^{s} \operatorname{sgn}\left(d_{i j}\right) \times\left(d_{i j}\right)^{2}=\sum_{k=1}^{s} \operatorname{sgn}\left(d_{k}\right) \times d_{k}^{2}=\prod_{k=1}^{n}\left(p_{k}^{2}-1\right)=\varphi(N) \psi(N)
$$

because $\left\{d_{i j}: 1 \leq j \leq s\right\}=\mathcal{S}$ by Lemma 3.3 (3).

- Assume that $i \neq j$. Then, $d_{i j} \neq N$. Let $q$ be a prime divisor of $N / d_{i j}$. We denote by $\mathcal{T}_{j}$ the subset of $\mathcal{S}$ such that

$$
\mathcal{T}_{j}:=\left\{d_{k} \in \mathcal{S}:\left(q, d_{k j}\right)=1\right\} .
$$

Then the size of $\mathcal{T}_{j}$ is $s / 2$. For each element $d_{k} \in \mathcal{T}_{j}$, we can find $d_{r(k)} \in \mathcal{S}$ such that $d_{r(k) j}=q \cdot d_{k j}$ by Lemma 3.3 (3). Moreover $\mathcal{T}_{j}^{c}=\left\{d_{r(k)}: d_{k} \in \mathcal{T}_{j}\right\}$ and we get $\operatorname{sgn}\left(d_{r(k) j}\right)=-\operatorname{sgn}\left(d_{k j}\right)$. For each $d_{k} \in \mathcal{T}_{j}$, we get $d_{i k} \times d_{k j}=d_{i r(k)} \times d_{r(k) j}$ by Lemma 3.3 (5). Therefore, we have

$$
B_{i j}=\sum_{k=1}^{s} \operatorname{sgn}\left(d_{k j}\right)\left(d_{i k} \times d_{k j}\right)=\sum_{d_{k} \in \mathcal{T}_{j}} \operatorname{sgn}\left(d_{k j}\right)\left[\left(d_{i k} \times d_{k j}\right)-\left(d_{i r(k)} \times d_{r(k) j}\right)\right]=0 .
$$

The matrix form of $C_{M, N}$ in the set $S_{2}$ on [10, P. 34] is then

$$
\text { for } 1 \leq a \leq s, \quad\left(C_{M, N}\right)_{a 1}=\left\{\begin{array}{cl}
(-1)^{\omega\left(d_{a}\right)}=(-1)^{n} \times \operatorname{sgn}\left(d_{a}\right) & \text { if } d_{a} \mid M, \\
0 & \text { otherwise. }
\end{array}\right.
$$

Finally, we prove the following lemma.

Lemma 3.6. Let $E:=\Lambda^{-1} C_{M, N}$. Then for $1 \leq a \leq s$ we have

$$
E_{a 1}=\operatorname{sgn}\left(d_{s+1-a}\right) \times \frac{24}{\varphi(N) \psi(N / M)} \times \frac{d_{s+1-a}}{\left(d_{s+1-a}, M\right)} .
$$

In particular, $E_{s 1}=(-1)^{\omega(N)} \frac{24}{\varphi(N) \psi(N / M)}$. Moreover if $M=N$, then we get

$$
E_{a 1}=\operatorname{sgn}\left(d_{s+1-a}\right) \times \frac{24}{\varphi(N)} .
$$

Proof. Let $D:=d_{s+1-a}=N / d_{a}$ and $E:=(D, M)$. Then, by direct calculation we have

$$
d_{a r}=d_{a} \boxplus d_{r}=\frac{D \times d_{r}}{\left(D, d_{r}\right)^{2}}
$$

and the sign of $\left(\Lambda^{-1}\right)_{a k} \times\left(C_{M, N}\right)_{k 1}$ is $\operatorname{sgn}\left(d_{a}\right) \times \operatorname{sgn}\left(d_{k}\right) \times(-1)^{n} \times \operatorname{sgn}\left(d_{k}\right)=\operatorname{sgn}(D)$ for any divisor $d_{k}$ of $M$. Therefore we have

$$
\begin{aligned}
\sum_{k=1}^{s} \operatorname{sgn}\left(d_{a k}\right) \times d_{a k} \times\left(C_{M, N}\right)_{k 1} & =\operatorname{sgn}(D) \times \sum_{d_{r} \mid M} \frac{D \times d_{r}}{\left(D, d_{r}\right)^{2}} \\
& =\operatorname{sgn}(D) \times \frac{D}{E} \times \sum_{d_{r} \mid M} \frac{E \times d_{r}}{\left(E, d_{r}\right)^{2}} .
\end{aligned}
$$


We denote by

$$
D_{r}:=\frac{E \times d_{r}}{\left(E, d_{r}\right)^{2}}=\frac{(D, M) \times d_{r}}{\left((D, M), d_{r}\right)^{2}} .
$$

Then, $D_{r}$ is a divisor of $M$ and for two distinct divisors $d_{r_{1}}, d_{r_{2}}$ of $M$, we get $D_{r_{1}} \neq D_{r_{2}}$. Therefore, we have

$$
\sum_{d_{r} \mid M} \frac{E \times d_{r}}{\left(E, d_{r}\right)^{2}}=\sum_{d_{r} \mid M} D_{r}=\sum_{d \mid M} d=\psi(M)
$$

which implies the result.

Now we give a proof of the theorem above.

Proof of Theorem 3.1. We check the conditions in Proposition 1 in op. cit. (We use the same notations.)

- The condition (0) implies that the order of $C_{M, N}$ is of the form $\frac{\varphi(N) \psi(N / M)}{24} \times g$ for some integer $g \geq 1$.

- The condition (1) always holds unless $M=N$ because $\sum_{\delta \mid N} r_{\delta} \cdot \delta=0$. If $M=N$, then $\sum_{\delta \mid N} r_{\delta} \cdot \delta=$ $(-1)^{n} g \varphi(N) \equiv 0(\bmod 24)$.

- The condition (2) implies that $g=\operatorname{num}\left(\frac{24}{\varphi(N) \psi(N / M)}\right) \times h$ for some integer $h \geq 1$ because $\sum_{\delta \mid N} r_{\delta} \cdot N / \delta=$ $g \varphi(N) \psi(N / M) \equiv 0(\bmod 24)$.

- The condition (3) always holds.

- The condition (4) always holds unless $M$ is a prime because $\prod_{\delta \mid N} \delta^{r_{\delta}}=1$. If $M$ is a prime, then it implies that $g \varphi(N / M)$ is even because $\prod_{\delta \mid N} \delta^{r_{\delta}}=M^{-g \varphi(N / M)}$.

In conclusion, the order of $C_{M, N}$ is equal to $\operatorname{num}\left(\frac{\varphi(N) \psi(N / M)}{24}\right) \times h$ for the smallest positive integer $h$ satisfying all the conditions above. Therefore we get $h=1$ unless all the following conditions hold:

(1) $M$ is a prime;

(2) $\varphi(N / M)=1$

(3) $\operatorname{num}\left(\frac{24}{\varphi(N) \psi(N / M)}\right)$ is odd.

Moreover if all the conditions above hold, then $h=2$. By the first condition, $M$ is a prime. By the second condition, either $N=M$ or $N=2 M$.

- Assume that $N=M$ is a prime greater than 3 . Then, $h=2$ if and only if $M \equiv 1(\bmod 8)$. This is proved by Ogg [14].

- Assume that $N=2 M$. Then, $h=2$ if and only if $M \equiv 1(\bmod 8)$. This is proved by Chua and Ling [1].

\section{EISENSTEIN SERIES}

As before, let $N=\prod_{i=1}^{n} p_{i}$ and $M=\prod_{i=1}^{m} p_{i}$ for $1 \leq m \leq n$. Let

$$
e(z):=1-24 \sum_{n \geq 1} \sigma(n) \times q^{n}
$$

be the $q$-expansion of Eisenstein series of weight 2 of level 1 as on [12, p. 78], where $\sigma(n)=\sum_{d \mid n} d$ and $q=e^{2 \pi i z}$.

Definition 4.1. For any modular form $g$ of weight $k$ and level $A$; and a prime $p$ not dividing $A$, we define modular forms $[p]_{k}^{+}(g)$ and $[p]_{k}^{-}(g)$ of weight $k$ and level $p A$ by

$$
[p]_{k}^{+}(g)(z):=g(z)-p^{k-1} g(p z) \quad \text { and } \quad[p]_{k}^{-}(g)(z):=g(z)-g(p z) .
$$

Using these operators, we define Eisenstein series of weight 2 and level $N$ by

$$
\mathcal{E}_{M, N}(z):=\left[p_{n}\right]_{2}^{-} \circ \cdots \circ\left[p_{m+1}\right]_{2}^{-} \circ\left[p_{m}\right]_{2}^{+} \circ \cdots \circ\left[p_{1}\right]_{2}^{+}(e)(z) .
$$

(Note that $\mathcal{E}_{M, N}=-24 E_{M, N}$, where $E_{M, N}$ is a normalized Eisenstein series in [21, §2.2].)

By Proposition 2.6 of op. cit., we know that $\mathcal{E}_{M, N}$ is an eigenform for all Hecke operators and $I_{M, N}$ annihilates $\mathcal{E}_{M, N}$. By Proposition 2.10 of op. cit., we can compute the residues of $\mathcal{E}_{M, N}$ at various cusps. 
Proposition 4.2. We have

$$
\operatorname{Res}_{P_{N}}\left(\mathcal{E}_{M, N}\right)=\left\{\begin{array}{cc}
(-1)^{n} \varphi(N) & \text { if } M=N \\
0 & \text { otherwise. }
\end{array}\right.
$$

Moreover, for a prime divisor p of $N$ we have

$$
\operatorname{Res}_{P_{N / p}}\left(\mathcal{E}_{N, N}\right)=(-1)^{n-1} \varphi(N) \quad \text { and } \operatorname{Res}_{P_{M}}\left(\mathcal{E}_{M, N}\right)=(-1)^{\omega(M)} \varphi(N) \psi(N / M)(M / N) .
$$

Proof. The first statement follows from the definition (cf. [12, §II.5]). For the second statement, we use the method of Deligne-Rapoport [3] (cf. 3.17 and 3.18 in $\S$ VII.3) or of Faltings-Jordan [6] (cf. Proposition 3.34). Therefore the residue of $\mathcal{E}_{M, N}$ at $P_{1}$ is $\varphi(N) \psi(N / M)(M / N)$ (cf. [21, Proposition 2.11]). Since the Atkin-Lehner operator $w_{p}$ acts by -1 on $\mathcal{E}_{M, N}$ for a prime divisor $p$ of $M, w_{M}$ acts by $(-1)^{\omega(M)}$ and hence the result follows.

\section{THE INDEX OF AN EISENSTEIN IDEAL}

As before, let $N=\prod_{i=1}^{n} p_{i}$ and $M=\prod_{i=1}^{m} p_{i}$ for some $1 \leq m \leq n$. Let $\mathbb{T}:=\mathbb{T}(N)$.

Note that $\mathbb{T} / I_{M, N} \simeq \mathbb{Z} / t \mathbb{Z}$ for some integer $t \geq 1$ [21, Lemma 3.1]. We compute the number $t$ as precise as possible.

Theorem 5.1. The index of $I_{N, N}$ is equal to the order of $C_{N, N}$ up to powers of 2.

Theorem 5.2. If $M \neq N$ and $N / M$ is odd (resp. even), then the index of $I_{M, N}$ and the order of $C_{M, N}$ coincide (resp. coincide up to powers of 2 ).

Before starting to prove the theorems, we introduce some notations.

Definition 5.3. For a prime $\ell$, we define $\alpha(\ell)$ and $\beta(\ell)$ as follows:

$$
\begin{gathered}
\left(\mathbb{T} / I_{M, N}\right) \otimes_{\mathbb{Z}} \mathbb{Z}_{\ell} \simeq \mathbb{Z} / \ell^{\alpha(\ell)} \mathbb{Z} \text { and } \\
\ell^{\beta(\ell)} \text { is the exact power of } \ell \text { dividing } \operatorname{num}\left(\frac{\varphi(N) \psi(N / M)}{24} \times h\right),
\end{gathered}
$$

where $h$ is the number in Theorem 3.1.

Since $I_{M, N}$ annihilates $C_{M, N}$, we get $\alpha(\ell) \geq \beta(\ell)$ (cf. [21, proof of Theorem 3.2]). Therefore to prove Theorems 5.1 and 5.2, it suffices to show that $\alpha(\ell) \leq \beta(\ell)$ for all (or odd) primes $\ell$. If $\alpha(\ell)=0$, then there is nothing to prove. Thus, we now assume that $\alpha(\ell) \geq 1$. Let

$$
\mathcal{I}:=\left(\ell^{\alpha(\ell)}, I_{M, N}\right)
$$

and let $\delta$ be a cusp form of weight 2 and level $N$ over the $\operatorname{ring} \mathbb{T} / \mathcal{I} \simeq \mathbb{Z} / \ell^{\alpha(\ell)} \mathbb{Z}$ whose $q$-expansion (at $P_{N}$ ) is

$$
\sum_{n \geq 1}\left(T_{n} \bmod \mathcal{I}\right) \times q^{n} .
$$

Now we prove the theorems above.

Proof of Theorem 5.1. First, let $\ell=3$ and $M=N$. Let $E:=\mathcal{E}_{N, N}\left(\bmod 3^{\alpha(3)+1}\right)$ and $A=(-1)^{\omega(N)} \varphi(N)$. Since $24 \delta$ is a cusp form of weight 2 modulo $3^{\alpha(3)+1}$ (cf. [12, p. 86]), $E+24 \delta$ is a modular form of weight 2 and level $N$ over $\mathbb{Z} / 3^{\alpha(3)+1} \mathbb{Z}$. Let $a=\min \{\alpha(3), \beta(3)+1\}$. Then, by the $q$-expansion principle [8, $\left.\S 1.6\right]$ we have

$$
E+24 \delta \equiv A e\left(\bmod 3^{a+1}\right)
$$

on the irreducible component $C$ of $X_{0}(N)_{\mathbb{F}_{\ell}}$ containing $P_{N}$ because $A e$ is a modular form of weight 2 over $\mathbb{Z} /(12 A) \mathbb{Z}$ and $\left(3^{\alpha(3)+1}, 12 A\right)=3^{a+1}$. By the following lemma, we get $A \equiv 0(\bmod 3)$ and hence we can choose a prime divisor $p$ of $N$ congruent to 1 modulo 3 . Note that the cusp $P_{N / p}$ belongs to $C$. By Proposition 4.2, $\operatorname{Res}_{P_{N / p}}(E)=-A$ and $\operatorname{Res}_{P_{N / p}}(A e) \equiv p A(\bmod 12 A)$ by Sublemma on [12, p. 86]. Combining all the computations above, we have

$$
\operatorname{Res}_{P_{N / p}}(8 \delta) \equiv \frac{(p+1) A}{3}\left(\bmod 3^{a}\right)
$$


Since $\delta$ is a cusp form modulo $3^{\alpha(3)}$, we get $\operatorname{Res}_{P_{N / p}}(8 \delta) \equiv 0\left(\bmod 3^{\alpha(3)}\right)$ and hence $3^{\beta(3)} \equiv 0\left(\bmod 3^{\alpha(3)}\right)$. In other words, we get $\alpha(3) \leq \beta(3)$.

Next, let $\ell \geq 5$ and $M=N$. Let $F:=\mathcal{E}_{N, N}\left(\bmod \ell^{\alpha(\ell)}\right)$. Then, $f:=F+24 \delta$ is a modular form of weight 2 and level $N$ over $\mathbb{Z} / \ell^{\alpha(\ell)} \mathbb{Z}$ whose $q$-expansion is $A$. Basically the inequality $\alpha(\ell) \leq \beta(\ell)$ follows from the non-existence of a $\bmod \ell$ modular form of weight 2 and leven $N$ whose $q$-expansion is a non-zero constant (cf. [12, chap. II, Proposition 5.6] and [13, Proposition (2.2.6)]).

- If $\ell \nmid N$, then by Ohta [13, Lemma (2.1.1)], we can find a modular form $g$ of weight 2 and level 1 such that $f(z)=g(N z)$. Therefore $A \equiv 0\left(\bmod \ell^{\alpha(\ell)}\right)($ cf. [12, chap. II, Proposition 5.6]) and hence we get $\alpha(\ell) \leq \beta(\ell)$.

- Assume that $\ell \mid N$ and $\mathfrak{m}:=(\ell, \mathcal{I})$ is not $\ell$-new. Then, the argument basically follows from the previous case because the exact powers of $\ell$ dividing $A$ and $\varphi(N / \ell)$ coincide. (For more detailed argument on lowering the level when $\ell \geq 5$, see the proof of Theorem 5.2 below.)

- Assume that $\ell \mid N$ and $\mathfrak{m}:=(\ell, \mathcal{I})$ is $\ell$-new. Then, we can lift $\delta$ to a modular form $\widetilde{\delta}$ of weight 2 and level $N$ over $\mathbb{Z}_{(\ell)}$ satisfying $w_{\ell}(\widetilde{\delta})=-\widetilde{\delta}$, where $\mathbb{Z}_{(\ell)}$ is the localization of $\mathbb{Z}$ at $\ell$. Therefore $\widetilde{\delta}$ determines a regular differential on $X_{0}(N)_{\mathbb{Z}_{(\ell)}}$ over $\mathbb{Z}_{(\ell)}$ (cf. [13, Proposition (1.4.9)]). Similarly, we can lift $F$ to $\mathcal{E}_{N, N}$ as well and $w_{\ell}\left(\mathcal{E}_{N, N}\right)=-\mathcal{E}_{N, N}$. Therefore $f=\mathcal{E}_{N, N}+24 \widetilde{\delta}\left(\bmod \ell^{\alpha(\ell)}\right)$ can be regarded as a regular differential on $X_{0}(N)_{\mathbb{Z}_{(\ell)}}$ over $\mathbb{Z} / \ell^{\alpha(\ell)} \mathbb{Z}$ whose $q$-expansion is $A$. If $\alpha(\ell) \geq \beta(\ell)+1$, then $g=f\left(\bmod \ell^{\beta(\ell)+1}\right)$ is a regular differential over $\mathbb{Z} / \ell^{\beta(\ell)+1} \mathbb{Z}$. Moreover $\ell^{-\beta(\ell)} \times g$ can be regarded as a regular differential over $\mathbb{F}_{\ell}$ whose $q$-expansion is a non-zero constant (cf. [12, p. 86]), which is a contradiction (cf. [13, Proposition (2.2.6)]). Thus, we get $\alpha(\ell) \leq \beta(\ell)$.

Lemma 5.4. If $\mathfrak{m}:=\left(3, I_{N, N}\right)$ is maximal, then $A=(-1)^{\omega(N)} \varphi(N) \equiv 0(\bmod 3)$.

Proof. As above, let $E:=\mathcal{E}_{N, N}(\bmod 9)$ and $\eta:=\delta(\bmod \mathfrak{m})$. Let $f:=E+24 \eta$ be a modular form of weight 2 and level $N$ over $\mathbb{Z} / 9 \mathbb{Z}$ whose $q$-expansion is $A$.

First, assume that 3 does not divide $N$. Then by Ohta [13, Lemma (2.1.1)], we can find a modular form $g$ of weight 2 and level 1 over $\mathbb{Z} / 9 \mathbb{Z}$ such that $f(z)=g(N z)$. By Mazur [12, chap. II, Proposition 5.6], we get $A \equiv 0(\bmod 3)$.

Next, assume that $p_{1}=3$ and $N=3 M$. If $\mathfrak{m}$ is 3 -old, then the result follows from the previous case. Thus, we further assume that $\mathfrak{m}$ is 3 -new. Then as above, we can regard $\eta$ as a regular differential on $X_{0}(N)_{\mathbb{Z}_{(\ell)}}$ over $\mathbb{F}_{3}$ and hence there is a modular form $\zeta$ of weight $3+1$ and level $M$ over $\mathbb{F}_{3}$ which has the same $q$-expansion as $\eta$ by Ohta [13, Proposition (2.2.4)]. By the same argument as on [12, p. 86], 240 $\zeta$ is a modular form of weight 4 and level $M$ over $\mathbb{Z} / 9 \mathbb{Z}$. Let $E_{4}$ be the usual Eisenstein series of weight 4 and level 1:

$$
E_{4}(z)=1+240 \sum_{n=1}^{\infty} \sigma_{3}(n) \times q^{n},
$$

where $\sigma_{3}(n)=\sum_{d \mid n} d^{3}$ and $q=e^{2 \pi i z}$. Let $G(z):=\left[p_{n}\right]_{4}^{+} \circ \cdots \circ\left[p_{2}\right]_{4}^{+}\left(E_{4}\right)(z)$ be an Eisenstein series of weight 4 and level $M$ whose constant term is $\prod_{i=2}^{n}\left(1-p_{i}^{3}\right)$. Now we consider the modular form $h:=G(\bmod 9)-240 \zeta$ of weight 4 and level $M$ over $\mathbb{Z} / 9 \mathbb{Z}$. Since the $q$-expansion of $h$ is $\prod_{i=2}^{n}\left(1-p_{i}^{3}\right)$, there is a modular form $H$ of weight 4 and level 1 over $\mathbb{Z} / 9 \mathbb{Z}$ such that $h(z)=H(M z)$ by Ohta [13, Lemma (2.1.1)]. However if $A \not \equiv 0(\bmod 3)$, then there is no such a modular form over $\mathbb{Z} / 9 \mathbb{Z}$ (cf. [13, p. 308]) because $1-p_{i}^{3} \equiv 1-p_{i}(\bmod 3)$. Therefore we get $A \equiv 0(\bmod 3)$.

Proof of Theorem 5.2. Since we assume that $\alpha(\ell) \geq 1, \mathfrak{m}:=\left(\ell, I_{M, N}\right)$ is maximal.

First, assume that $N / M$ is divisible by an odd prime $\ell$. Then $U_{\ell} \equiv \ell \equiv 0(\bmod \mathfrak{m})$ and hence $\mathfrak{m}$ is not $\ell$ new. Thus, we get $\mathbb{T}(N) / \mathcal{I} \simeq \mathbb{T}(N)^{\ell \text {-old }} / \mathcal{I}$. Let $R$ be the common subring of $\mathbb{T}(N / \ell)$ and $\mathbb{T}(N)^{\ell \text {-old }}$, which is generated by all $T_{n}$ with $\ell \nmid n$. Then, as in the proof of Lemma $2.1, \mathbb{T}(N / \ell)=R\left[T_{\ell}\right]$ and $\mathbb{T}(N)^{\ell \text {-old }}=R\left[U_{\ell}\right]$. Note that if $\ell$ is odd then $R=\mathbb{T}(N / \ell)$ by Ribet [20, p. 491] and $\mathbb{T}(N)^{\ell \text {-old }} \simeq R[X] /\left(X^{2}-T_{\ell} X+\ell\right)$. Let $I$ be the ideal of $R$ generated by all the generators of $\mathcal{I}$ but $U_{\ell}-\ell$. Then, we show that $T_{\ell}-\ell-1 \in I$ as follows. Note 
that the kernel $K$ of the composition of the maps

$$
R=\mathbb{T}(N / \ell) \hookrightarrow \mathbb{T}(N)^{\ell \text {-old }}=R\left[U_{\ell}\right] /\left(U_{\ell}^{2}-T_{\ell} U_{\ell}+\ell\right) \rightarrow \mathbb{T}(N)^{\ell \text {-old }} / \mathcal{I} \simeq \mathbb{Z} / \ell^{\alpha(\ell)} \mathbb{Z}
$$

(sending $T_{n}$ to $\left.T_{n}(\bmod \mathcal{I})\right)$ is $\left(I, \ell\left(T_{\ell}-\ell-1\right)\right)$ and this composition is clearly surjective. Thus, we get $R / I \rightarrow$ $R / K$. Since all the generators of $R$ are congruent to integers modulo $I$; and $I$ contains $\ell^{\alpha(\ell)}$, we have $R / I=$ $R / K \simeq \mathbb{Z} / \ell^{\alpha(\ell)} \mathbb{Z}$; in particular $\ell\left(T_{\ell}-\ell-1\right) \in I$. Let $f$ be a cusp form over $R / I$ whose $q$-expansion is $\sum_{n \geq 1}\left(T_{n} \bmod I\right) \times q^{n}$.

- Suppose that $\ell \geq 5$. Let $E:=\mathcal{E}_{M, N / \ell}\left(\bmod \ell^{\alpha(\ell)}\right)$ and let $g:=24 f+E$. Then, $g$ is a modular form over $R / I \simeq \mathbb{Z} / \ell^{\alpha(\ell)} \mathbb{Z}$ whose $q$-expansion is of the form $\sum_{k \geq 0} a_{k} \times q^{\ell k}$. By Katz [9, Corollaries (2) and (3) of the main theorem], we get $g=0$ and hence $a_{1} / 24=T_{\ell}-\ell-1 \in I$. (Note that the constant term $a_{0}$ must be 0 and hence we get $\alpha(\ell) \leq \beta(\ell)$ as well if $M=N / \ell$.)

- Suppose that $\ell=3$. Let $E:=\mathcal{E}_{M, N / \ell}\left(\bmod 3^{\alpha(3)+1}\right)$ and let $g:=24 f+E$. Then, $g$ is a modular form over $\mathbb{Z} / 3^{\alpha(3)+1} \mathbb{Z}$ whose $q$-expansion is of the form $\sum_{k \geq 0} a_{k} \times q^{\ell k}$. If $a_{1}=0 \in \mathbb{Z} / 3^{\alpha(3)+1} \mathbb{Z}$ then $a_{1} / 24=T_{3}-4=0 \in \mathbb{Z} / 3^{\alpha(3)} \mathbb{Z} \simeq R / I$ and hence $T_{3}-4 \in I$. Therefore it suffices to show that $a_{1}=0 \in \mathbb{Z} / 3^{\alpha(3)+1} \mathbb{Z}$.

If $M \neq N / \ell$ then $a_{0}=0$ and hence $g=0$ by Corollaries (3) and (4) in loc. cit. Therefore $a_{1}=0$.

Suppose that $M=N / \ell$. Then, $a_{0}=(-1)^{\omega(M)} \varphi(M)$. Note that the exact power of 3 dividing $a_{0}$ is $3^{\beta(3)+1}$. Since $3\left(T_{3}-4\right) \in I, g\left(\bmod 3^{\alpha(3)}\right)$ is a modular form over $\mathbb{Z} / 3^{\alpha(3)} \mathbb{Z}$ whose $q$-expansion is a constant $a_{0}$. Since $a_{0} \times e$ is a modular form over $\mathbb{Z} / 3^{\beta(3)+2} \mathbb{Z}$ whose $q$-expansion is $a_{0}$, by the $q$-expansion principle $g=24 f+E \equiv a_{0} \times e\left(\bmod 3^{a}\right)$, where $a=\min \{\alpha(3), \beta(3)+2\}$. Since $\mathfrak{m}$ is $\ell$-old, there is the corresponding maximal ideal $\mathfrak{n}$ of $\mathbb{T}(N / \ell)$ to $\mathfrak{m}$. Hence by Lemma $5.4, a_{0} \equiv 0(\bmod 3)$ and we can find a prime divisor $p$ of $N / \ell$ such that $p \equiv 1(\bmod 3)$. By comparing the residues of $g$ and $a_{0} \times e$ at $P_{N / p}$ as in the proof of Theorem 5.1, we get $(p+1) a_{0} \equiv 0\left(\bmod 3^{a}\right)$ and hence $\alpha(3) \leq \beta(3)+1$. Therefore $h:=3^{-\alpha(3)} \times g$ is a modular form over $\mathbb{F}_{3}$. Again by Corollary (5) in loc. cit. and by Mazur [12, Proposition $5.6(\mathrm{~b})]$, we get $h=3^{-\alpha(3)} \times a_{0} \times e$; in particular, $3^{-\alpha(3)} \times a_{1} \equiv 0(\bmod 3)$, i.e., $a_{1}=0 \in \mathbb{Z} / 3^{\alpha(3)+1} \mathbb{Z}$ as desired.

(Note that in the first case, we can allow the case where $M=N$ by taking $E:=\mathcal{E}_{M / \ell, N / \ell}\left(\bmod \ell^{\alpha(\ell)}\right)$, which is used in the proof of Theorem 5.1 above.) Therefore we have $I=\left(\ell^{\alpha(\ell)}, I_{M, N / \ell}\right)$ and

$$
\mathbb{T}(N) / \mathcal{I} \simeq \mathbb{T}(N)^{\ell \text {-old }} / \mathcal{I} \simeq R / I=\mathbb{T}(N / \ell) /\left(\ell^{\alpha(\ell)}, I_{M, N / \ell}\right)
$$

Accordingly, it suffices to prove that $\alpha(\ell) \leq \beta(\ell)$ for primes $\ell$ not dividing $N / M$ because $\ell \nmid \ell^{2}-1$.

Next, we assume that $\ell$ does not divide $N / M$. Let $F:=\mathcal{E}_{M, N}\left(\bmod 24 \ell^{\alpha(\ell)}\right)$ and $\delta$ be a cusp form as above. Since $F$ and $-24 \delta$ have the same $q$-expansions (at $P_{N}$ ), they coincide on the irreducible component $D$ of $X_{0}(N)_{\mathbb{F}_{\ell}}$, which contains $P_{N}$. Note that the cusp $P_{M}$ belongs to $D$ because $\ell \nmid N / M$. Since $-24 \delta$ is a cusp form over the ring $\mathbb{Z} / 24 \ell^{\alpha(\ell)} \mathbb{Z}$, the residue of $F$ at $P_{M}$ must be zero. By Proposition 4.2, $\varphi(N) \psi(N / M)(M / N) \equiv 0\left(\bmod 24 \ell^{\alpha(\ell)}\right)$. Therefore we get $\alpha(\ell) \leq \beta(\ell)$. (Note that if $\ell=2$, then $h=1$ with the assumption that $M \neq N$ and $\ell \nmid N / M$.)

If $\ell$ is odd and $\ell \nmid \varphi(N)$, we prove the following.

Proposition 5.5. Let $\ell$ be an odd prime and $\mathfrak{m}:=\left(\ell, I_{1, N}\right)$. Hence, we assume that $\ell \nmid \varphi(N)$ from the definition (cf. §2). Then, $\mathfrak{m}$ cannot be maximal.

Proof. Assume that $\mathfrak{m}$ is maximal. If $\ell \mid N$, then $\mathfrak{m}$ cannot be $\ell$-new because $U_{\ell} \equiv \ell \equiv 0(\bmod \mathfrak{m})$. Therefore there is a maximal ideal $\mathfrak{n}:=\left(\ell, I_{1, N / \ell}\right)$ in the Hecke $\operatorname{ring} \mathbb{T}(N / \ell)$ of level $N / \ell$. Thus, we may assume that $\ell \nmid N$. Then as above, $\delta$ is a $\bmod \ell$ cusp form of weight 2 and level $N$. Let $g=\mathcal{E}_{N, N}(\bmod 24 \ell)+24 \delta$ be a modular form over $\mathbb{Z} / 24 \ell \mathbb{Z}$.

First, consider the case where $n=\omega(N)=1$.

- If $\ell \geq 5$, then $g$ is a $\bmod \ell$ modular form of weight 2 and level $N$ as above. Since the $q$-expansion of $g$ is

$$
(1-N)+24(1-N) \sum_{i=1}^{\infty} \sigma(d) \times q^{d N},
$$


we get $\frac{g}{1-N}=0$ by Mazur [12, chap. II, Corollary 5.11], which is a contradiction. Therefore $\mathfrak{m}$ is not maximal.

- If $\ell=3$, then $g$ is a modular form of weight 2 and level $N$ over $\mathbb{Z} / 9 \mathbb{Z}$ as above. Then, by Mazur [12, chap. II, Lemma 5.9], there is a modular form $G$ of level 1 over $\mathbb{Z} / 9 \mathbb{Z}$ such that $G(N z)=\frac{g(z)}{1-N}$. However this contradicts Proposition 5.6(c) in [12, chap. II]. Therefore $\mathfrak{m}$ is not maximal.

Next, consider the case where $n \geq 2$. Let $F_{N}(q):=(-1 / 24) \times \mathcal{E}_{1, N} \in \mathbb{Z}[[q]]$ be a formal $q$-expansion. Since $\mathfrak{m}$ is maximal, $\delta \equiv F_{N}(q)(\bmod \ell)$ is a $\bmod \ell$ modular form of weight 2 and level $N$. Then, by the following lemma, we can lower the level of $\delta$ because $\varphi(N) \not \equiv 0(\bmod \ell)$. Therefore the result follows from the case where $n=1$.

Lemma 5.6. Let $N=p D$ be a square-free integer with $D>1$ and $p$ a prime. Assume that $p \not \equiv 1(\bmod \ell)$ and $\ell \nmid N$. Let $F_{N}(q):=(-1 / 24) \times \mathcal{E}_{1, N} \in \mathbb{Z}[[q]]$ be a formal q-expansion. If $F_{N}(q)(\bmod \ell)$ is the q-expansion of a mod $\ell$ modular form of weight 2 and level $N$, then $F_{D}(q)(\bmod \ell)$ is also the q-expansion of a mod $\ell$ modular form of weight 2 and level $D$.

Proof. Let $G(q):=(-1 / 24) \times \mathcal{E}_{p, N}$. Then, as formal $q$-expansions we get

$$
F_{N}(q)-G(q)=(p-1) F_{D}\left(q^{p}\right) .
$$

Therefore if $F_{N}(q)(\bmod \ell)$ is the $q$-expansion of a $\bmod \ell$ modular form of level $N$, then there is a $\bmod \ell$ modular form of level $D$ whose $q$-expansion is $(p-1) F_{D}(q)(\bmod \ell)$ by Ohta [13, Lemma (2.1.1)]. Therefore the result follows because $p \not \equiv 1(\bmod \ell)$.

\section{PROOF OF THE MAIN THEOREM}

In this section, we prove our main theorem.

Theorem 6.1. Let $\mathfrak{m}:=\left(\ell, I_{M, N}\right)$ be a maximal ideal of $\mathbb{T}(N)$. Then $\mathcal{C}_{N}[\mathfrak{m}] \neq 0$.

Proof. If $\ell$ is odd, then the result follows from Theorems 5.1 and 5.2. Therefore we assume that $\ell=2$. By the definition of the notation, $M$ is either $N$ or $N / 2$.

- If $N$ is a prime and $N=M$, then $M \equiv 1(\bmod 8)$ by Mazur [12]. Thus, we have $\mathcal{C}_{N}[\mathfrak{m}] \neq 0$.

- If $N$ is not a prime and $N=M$, then we set $N=p D$ with $D$ odd and $\omega(D) \geq 1$. (In other words, if $N$ is even then we set $p=2$.) Since $\left(2, I_{N, N}\right)=\left(2, I_{p, N}\right)$ is maximal, the index of $I_{p, N}$, which is equal to the order of $C_{p, N}$, is divisible by 2 and hence $\left\langle C_{p, N}\right\rangle[\mathfrak{m}] \neq 0$, which implies that $\mathcal{C}_{N}[\mathfrak{m}] \neq 0$.

- If $N=2 M$ with $\omega(M)=1$, then $\mathfrak{m}$ is not 2-new and hence there is the corresponding Eisenstein maximal ideal of $\mathbb{T}(M)$. Therefore $M \equiv 1(\bmod 8)$ by Mazur. This implies that the order of $C_{M, N}$ is $\frac{M-1}{4}$ by Theorem 3.1. Thus, we get $\mathcal{C}_{N}[\mathfrak{m}] \neq 0$.

- If $N=2 M$ with $\omega(M) \geq 2$, then the order of $C_{p, N}$ is divisible by 2 , where $p$ is any prime divisor of $M$. Therefore we get $\mathcal{C}_{N}[\mathfrak{m}] \neq 0$.

\section{REFERENCES}

[1] Seng-Kiat Chua and San Ling, On the rational cuspidal subgroup and the rational torsion points of $J_{0}(p q)$, Proc. Amer. Math. Soc., Vol 125, Number 8 (1997), 2255-2263.

[2] Henry Darmon and Fred Diamond and Richard Taylor, Fermat's Last Theorem, Elliptic curves, modular forms and Fermat's last theorem (Hong Kong, 1993), International press, Cambridge (1995), 2-140.

[3] Pierre Deligne and Michael Rapoport, Les schémas de modules de courbes elliptiques, Modular functions of one variable II, Lecture notes in Math., Vol. 349 (1973), 143-316.

[4] Vladimir Drinfeld, Two theorems on modular curves, Functional Anal. Appl. 7 (1973), 155-156.

[5] Bas Edixhoven, The weight in Serre's conjectures on modular forms, Invent. Math. 109 (1992), 563-594.

[6] Gerd Faltings and Bruce Jordan, Crystalline cohomology and GL(2, $\mathbb{Q})$, Israel Journal of Math., Vol 90. (1995), 1-66.

[7] Günter Harder, Eisenstein cohomology and the construction of mixed motives, preprint, available at http://www.math.uni-bonn.de/people/harder/Manuscripts/Eisenstein/Mix-Mot-2015.pdf(2015). 
[8] Nicholas Katz, p-adic properties of modular schemes and modular forms, Modular functions of one variable III, Lecture notes in Math., Vol. 350 (1973), 69-190.

[9] Nicholas Katz, A result on modular forms in characteristic p, Modular functions of one variable V, Lecture notes in Math., Vol. 601 (1976), 53-61.

[10] San Ling, On the $\mathbb{Q}$-rational cuspidal subgroup and the component group of $J_{0}\left(p^{r}\right)$, Israel Journal of Math., Vol 99 (1997), $29-54$.

[11] Yuri Manin, Parabolic points and zeta functions of modular curves (in Russian), Izv. Akad. Nauk SSSR Ser. Mat., 36, 19-66 (1972). Translation in Math USSR-Izv 6 (1972), 19-64.

[12] Barry Mazur, Modular curves and the Eisenstein Ideal, Publications Math. de l'I.H.É.S., tome 47 (1977), $33-186$.

[13] Masami Ohta, Eisenstein ideals and the rational torsion subgroups of modular Jacobian varieties II, Tokyo Journal of Math., Vol. 37, no. 2 (2014), 273-318.

[14] Andrew Ogg, Rational points on certain elliptic modular curves, Proc. Sympos. Pure Math., vol. 24, AMS, Providence, R. I. (1973), 221-231.

[15] Andrew Ogg, Hyperelliptic modular curves, Bull. Soc. Math. France, Vol. 102 (1974), 449-462.

[16] Kenneth Ribet, Congruence relations between modular forms, Proceeding of the International Congress of Math., Vol. 1, 2 (Warsaw, 1983) (1983), 503-514.

[17] Kenneth Ribet, On modular representations of $\mathrm{Gal}(\overline{\mathbb{Q}} / \mathbb{Q})$ arising from modular forms, Invent. Math. 100, no. 2 (1990), 431-476.

[18] Kenneth Ribet, Eisenstein primes for $J_{0}(p q)$, unpublished (2008).

[19] Toshikazu Takagi, The cuspidal class number formula for the modular curves $X_{0}(M)$ with $M$ square-free, Journal of Algebra, Vol. 193 (1997), 180-213.

[20] Andrew Wiles, Modular elliptic curves and Fermat's Last Theorem, Annals of Math., Vol. 141 (1995), $443-551$.

[21] Hwajong Yoo, The index of an Eisenstein ideal and multiplicity one, submitted, available at http: / /arxiv.org/pdf/1311.5275.pdf(2014).

[22] Hwajong Yoo, Non-optimal levels of a reducible mod $\ell$ modular representation, submitted, available at http://arxiv.org/pdf/1409.8342.pdf (2014).

Center for Geometry and Physics, Institute for Basic Science (IBS), Pohang, Republic of Korea 37673

E-mail address: hwajong@gmail.com 